\title{
Projection Methods for a System of Nonlinear Mixed Variational Inequalities in Banach Spaces
}

\author{
Zhong-Bao Wang, ${ }^{1}$ Guo-Ji Tang, ${ }^{2}$ and Hong-Ling Zhang ${ }^{1}$ \\ ${ }^{1}$ Department of Mathematics, Southwest Jiaotong University, Chengdu 610031, China \\ ${ }^{2}$ School of Science, Guangxi University for Nationalities, Nanning, Guangxi 530006, China \\ Correspondence should be addressed to Zhong-Bao Wang; zhongbaowang@hotmail.com
}

Received 29 October 2013; Accepted 8 December 2013; Published 15 May 2014

Academic Editor: M. Van Nguyen

Copyright (c) 2014 Zhong-Bao Wang et al. This is an open access article distributed under the Creative Commons Attribution License, which permits unrestricted use, distribution, and reproduction in any medium, provided the original work is properly cited.

The existence and uniqueness of solution for a system of nonlinear mixed variational inequality in Banach spaces is given firstly. A Mann iterative sequences with errors for this system of nonlinear mixed variational inequalities in Banach spaces is studied, by using the generalized $f$-projection operator $\pi_{K}^{f}$. Our results extend the main results in (Verma (2005); Verma (2001)) from Hilbert spaces to Banach spaces.

\section{Introduction}

Throughout this paper, unless otherwise stated, we let $B$ be a real Banach space with the norm $\|\cdot\|$, we let $B^{*}$ be the topological dual space of $B$, we let $\langle\cdot, \cdot\rangle$ be the duality between $B$, and we let $B^{*}$ and $K \subset B$ be a nonempty closed convex set.

Let $A_{1}, A_{2}, S_{1}, S_{2}: K \rightarrow B^{*}$ be single-valued mappings. Let $J: B \rightarrow B^{*}$ be the normalized duality mapping, and let $f: K \subset B \rightarrow R \cup\{+\infty\}$ be a proper lower semicontinuous mapping. We will consider the following system of nonlinear mixed variational inequalities problems (SNMVIP): find $\left(x^{*}, y^{*}\right) \in K \times K$ satisfying

$$
\begin{aligned}
& \left\langle\rho_{1} A_{1}\left(y^{*}\right)+\rho_{1} S_{1}\left(y^{*}\right)+J\left(x^{*}\right)-J\left(y^{*}\right), x-x^{*}\right\rangle \\
& +f(x)-f\left(x^{*}\right) \geq 0, \quad \forall x \in K, \\
& \left\langle\eta_{1} A_{2}\left(x^{*}\right)+\eta_{1} S_{2}\left(x^{*}\right)+J\left(y^{*}\right)-J\left(x^{*}\right), x-y^{*}\right\rangle \\
& +f(x)-f\left(y^{*}\right) \geq 0, \quad \forall x \in K,
\end{aligned}
$$

where $\rho_{1}>0$ and $\eta_{1}>0$.
If $B=H$ is a real Hilbert space, $S_{1}=S_{2}=S, A_{1}=$ $A_{2}=0$, and $f=0$, then SNMVIP (1) reduces to the following problem:

$$
\begin{array}{ll}
\left\langle\rho_{1} S\left(y^{*}\right)+x^{*}-y^{*}, x-x^{*}\right\rangle \geq 0, & \forall x \in K, \\
\left\langle\eta_{1} S\left(x^{*}\right)+y^{*}-x^{*}, x-y^{*}\right\rangle \geq 0, & \forall x \in K .
\end{array}
$$

The problem (2) was introduced and studied by Verma [1, $2]$. We note that for $\rho_{1}=0$ the problem (2) reduces to the nonlinear variational inequalities problem: determine an element $x^{*} \in K$ such that

$$
\left\langle S\left(x^{*}\right), x-y^{*}\right\rangle \geq 0, \quad \forall x \in K .
$$

In brief, the system of nonlinear mixed variational inequalities (1) is more general and includes many systems of variational inequalities and variational inequalities as special cases.

It is well known that the projection method and its variant forms have represented an important tool for solving variational inequalities and the system of variational inequalities; see [1-21] and the references therein. Wu and Huang [10] introduced and studied a new class of generalized $f$ projection operators in Banach spaces, which extends the 
definition of the generalized projection operators introduced and studied by Alber [3, 4] and Li [7]. Some properties of the generalized $f$-projection operator are given in [10]. By using the generalized projection operators and the generalized $f$-projection operators, some authors studied the existence theorems of solution and some iterative algorithms of approximating solutions for variational inequalities; see $[3,4,6-8,10-12]$.

In addition, Verma $[1,2]$ applies the metric projection operator technique and the fixed theorem to suggest some iterative algorithms for the system of variational inequalities with the monotone mappings in Hilbert spaces. By using the sunny nonexpansive retraction, Yao et al. [21] prove that the suggested two-step projection method converges strongly to the solution of a system of variational inequality, which extend the main results in Verma [1] from Hilbert spaces to Banach spaces. Recently, Wang et al. show that the Lipschitz continuity of the generalized $f$-projection operator and the normalized duality mapping and study a class of system of generalized mixed variational inequalities in Banach spaces.

Motivated and inspired by the research work going on this field, in this paper, we firstly show the existence and uniqueness of solution for a system of nonlinear mixed variational inequality in Banach spaces. By using the generalized $f$-projection operator $\pi_{K}^{f}$, we study Mann iterative sequences with errors for this system of nonlinear mixed variational inequalities in Banach spaces. Our results extend and improve the main results in Verma $[1,2]$ from Hilbert spaces to Banach spaces.

\section{Preliminaries}

Recall that $B$ is said to be strictly convex if $\|(x+y) / 2\|<1$ for all $x, y \in B$ with $\|x\|=\|y\|=1$ and $x \neq y$. It is also said to be uniformly convex if $\lim _{n \rightarrow \infty}\left\|x_{n}-y_{n}\right\|=0$ for any two sequences $\left\{x_{n}\right\},\left\{y_{n}\right\}$ in $B$ with $\left\|x_{n}\right\|=\left\|y_{n}\right\|=1$ and $\lim _{n \rightarrow \infty}\left\|\left(x_{n}+y_{n}\right) / 2\right\|=1$. The function

$$
\delta_{B}(\epsilon)=\inf \left\{1-\frac{\|x+y\|}{2}:\|x\|=1,\|y\|=1,\|x-y\| \geq \epsilon\right\}
$$

is called the modulus of convexity of $B$. It is well known that $B$ is 2-uniformly convex if and only if there exists a constant $k_{B}>0$ such that $\delta(\epsilon) \geq k_{B} \epsilon^{2}$ for all $\epsilon \in(0,2]$. Recall that $B$ is said to be smooth if $\lim _{t \rightarrow 0}(\|x+t y\|-\|x\|) / t$ exists for all $x, y \in B$ with $\|x\|=\|y\|=1$. It is also said to be uniformly smooth if the limit is attained uniformly for $\|x\|=\|y\|=1$. The function

$$
\rho_{B}(t)=\sup \left\{\frac{\|x+y\|+\|x-y\|}{2}-1:\|x\|=1,\|y\| \leq t\right\}
$$

is called the modulus of smoothness of $B$. It is known that $B$ is said to be 2-uniformly smooth if and only if there exists a constant $c_{B}>0$ such that $\rho_{B}(t) \leq c_{B} t^{2}$. Moreover, $B^{*}$ is uniformly convex if and only if $B$ is uniformly smooth. In this case, $B$ is reflexive by the Milman theorem.
Next we recall the concept of the normalized duality mapping. The normalized duality mapping $J: B \rightarrow 2^{B^{*}}$ is defined by

$$
\begin{aligned}
J(x)=\left\{j(x) \in B^{*}:\langle j(x), x\rangle\right. & \\
& \left.=\|j(x)\|\|x\|=\|x\|^{2}=\|j(x)\|^{2}\right\}, \quad \forall x \in B .
\end{aligned}
$$

Many properties of the normalized duality mapping $J$ can be found in [18].

We list some properties of $J$ as follows:

$\left(a_{1}\right)$ if $B$ is a smooth Banach space, then $J$ is single-valued and continuous from the strong topology of $B$ to the weak $*$ topology of $B^{*}$;

$\left(a_{2}\right) J$ is the identity operator in Hilbert spaces;

$\left(a_{3}\right)$ if $B$ is a reflexive, smooth, and strictly convex Banach space, and $J^{*}: B^{*} \rightarrow B$ is the normalized duality mapping on $B^{*}$, then $J^{-1}=J^{*}, J J^{*}=I_{B^{*}}$ and $J^{*} J=$ $I_{B}$.

For any fixed $\rho>0$, let $G: B^{*} \times K \rightarrow(-\infty,+\infty]$ be a function defined as follows:

$$
\begin{array}{r}
G(\varphi, x)=\|x\|^{2}-2\langle\varphi, x\rangle+\|\varphi\|^{2}+2 \rho f(x), \\
\forall \varphi \in B^{*}, \quad \forall x \in K,
\end{array}
$$

where $\varphi \in B^{*}, x \in K$ and $f: K \subset B \rightarrow R \cup\{+\infty\}$ is proper, convex, and lower semicontinuous. It is easy to see that $G(\varphi, x) \geq(\|x\|-\|\varphi\|)^{2}+2 \rho f(x)$ for all $\varphi \in B^{*}$ and $x \in K$.

Definition 1 . We say that $B$ has the property $(h)$ if $x_{n} \rightarrow x$ weakly and $\left\|x_{n}\right\| \rightarrow\|x\|$ implies $x_{n} \rightarrow x$.

Remark 2. It is well known that any locally uniformly convex Banach space has the property $(h)$ and $B^{*}$ has a Fréchet differentiable norm if and only if $B$ is reflexive and strictly convex and has the property $(h)$ (see, e.g., [19]).

Definition 3 (see [10]). We say that $\pi_{K}^{f}: B^{*} \rightarrow 2^{K}$ is a generalized $f$-projection operator if

$$
\pi_{K}^{f} \varphi=\left\{u \in K: G(\varphi, u)=\inf _{y \in K} G(\varphi, y)\right\}, \quad \forall \varphi \in B^{*} .
$$

Remark 4. (i) If $f(x)=0$ for all $x \in K$, then the generalized $f$-projection operator $\pi_{K}^{f}$ reduces to the generalized projection operator $\pi_{k}$ defined by Alber [4] and Li [7]; that is,

$$
\pi_{K} \varphi=\left\{u \in K: G_{1}(\varphi, u)=\inf _{y \in K} G_{1}(\varphi, y)\right\}, \quad \forall \varphi \in B^{*},
$$

where $G_{1}(\varphi, x)=\|x\|^{2}-2\langle\varphi, x\rangle+\|\varphi\|^{2}$ for all $\varphi \in B^{*}$ and $x \in K$.

(ii) If $f(x)=0$ for all $x \in K$ and $B=\mathscr{H}$ is a Hilbert space, then the generalized $f$-projection operator $\pi_{K}^{f}$ is equivalent to the following metric projection operator:

$$
P_{K}(\varphi)=\left\{u \in K:\|u-\varphi\|=\inf _{y \in K}\|y-\varphi\|\right\}, \quad \forall \varphi \in \mathscr{H} .
$$


Definition 5. Let $B$ be a reflexive, smooth, and strictly convex Banach space with the dual space $B^{*}$, and $2^{B^{*}}$ denote the family of all nonempty subsets of $B^{*} . T: B \rightarrow 2^{B^{*}}$ is said to be $J$-strongly monotone if there exists a constant $S>0$ satisfying

$$
\begin{array}{r}
\left\langle u-v, J^{*}(J(x)-J(y))\right\rangle \geq S\|x-y\|^{2}, \\
\forall u \in T(x), \quad \forall v \in T(y) .
\end{array}
$$

Remark 6. If $B=\mathscr{H}$ is a Hilbert space, then $J$-strongly monotonicity reduce to strongly monotonicity in [15].

Theorem 7 (see $[6,10]$ ). If $B$ is a reflexive Banach space with dual space $B^{*}$ and $K$ is a nonempty closed convex subset of $B$, then the following conclusions hold:

(i) for any given $\varphi \in B^{*}, \pi_{K}^{f} \varphi$ is a nonempty, closed, and convex subset of $K$;

(ii) if $B$ is smooth, then for any given $\varphi \in B^{*}, x \in \pi_{K}^{f} \varphi$ if and only if

$\langle\varphi-J(x), x-y\rangle+\rho f(y)-\rho f(x) \geq 0, \quad \forall y \in K$

(iii) if $B$ is strictly convex, then the operator $\pi_{K}^{f}: B^{*} \rightarrow K$ is single-valued.

Lemma 8 (see [13]). B is 2-uniformly smooth if and only if there exists a constant $C_{B}>0$ such that, for all $x, y \in B$,

$$
\|x+y\|^{2} \leq\|x\|^{2}+2\langle y, J x\rangle+C_{B}\|y\|^{2} .
$$

Lemma 9 (see [20]). Let $\left\{a_{n}\right\},\left\{b_{n}\right\}$, and $\left\{c_{n}\right\}$ be three sequences of nonnegative numbers satisfying the following conditions: there exists $n_{0}$ such that

$$
a_{n+1} \leq\left(1-t_{n}\right) a_{n}+b_{n} t_{n}+c_{n}, \quad \forall n \geq n_{0},
$$

where

$$
\begin{aligned}
t_{n} \in[0,1], \quad \sum_{n=0}^{\infty} t_{n}=+\infty ; \quad \lim _{n \rightarrow \infty} b_{n}=0, \\
\\
\sum_{n=0}^{\infty} c_{n}<+\infty .
\end{aligned}
$$

Then $a_{n} \rightarrow 0$ as $n \rightarrow+\infty$

Proposition 10 (see [14]). Let $B$ be a reflexive, smooth, and strictly convex Banach space with the dual space $B^{*}$. Then for any $x, y \in B$,

$$
\langle J x-J y, x-y\rangle \geq 2 C_{1}^{2} \delta_{B}\left(\frac{\|x-y\|}{2 C_{1}}\right),
$$

where $C_{1}=\sqrt{\left(\|x\|^{2}+\|y\|^{2}\right) / 2}$.
Proposition 11 (see [14]). Let B be a real uniformly convex and uniformly smooth Banach space. Then

$$
\|J x-J y\| \leq 8 M_{2} h_{B}\left(\frac{4\|x-y\|}{M_{2}}\right), \quad \forall x, y \in B,
$$

where $M_{2}=\sqrt{\left(\|x\|^{2}+\|y\|^{2}\right) / 2}$ and $h_{B}(\tau)=\rho_{B}(\tau) / \tau$.

Proposition 12. Let $B$ be a reflexive, smooth, and strictly convex Banach space with the dual space $B^{*}$, let $B$ have the property ( $h)$, and let $K$ be a nonempty closed convex subset of B. For any $x_{1}^{*}, x_{2}^{*} \in B^{*}$, let $x_{1}=\pi_{K}^{f} x_{1}^{*}$ and $x_{2}=\pi_{K}^{f} x_{2}^{*}$. Then

$$
\left\langle x_{1}^{*}-x_{2}^{*}, x_{1}-x_{2}\right\rangle \geq 2 C_{1}^{2} \delta_{B}\left(\frac{\left\|x_{1}-x_{2}\right\|}{2 C_{1}}\right),
$$

where $C_{1}=\sqrt{\left(\left\|x_{1}\right\|^{2}+\left\|x_{2}\right\|^{2}\right) / 2}$.

Proof. According to Proposition 10,

$$
\left\langle J x_{1}-J x_{2}, x_{1}-x_{2}\right\rangle \geq 2 C_{1}^{2} \delta_{B}\left(\frac{\left\|x_{1}-x_{2}\right\|}{2 C_{1}}\right),
$$

where $C_{1}=\sqrt{\left(\left\|x_{1}\right\|^{2}+\left\|x_{2}\right\|^{2}\right) / 2}$. Since $x_{1}=\pi_{K}^{f} x_{1}^{*}$ and $x_{2}=$ $\pi_{K}^{f} x_{2}^{*}$, Theorem 7 yields

$$
\begin{aligned}
& \left\langle J x_{1}-x_{1}^{*}, x_{2}-x_{1}\right\rangle+\rho f\left(x_{2}\right)-\rho f\left(x_{1}\right) \geq 0, \\
& \left\langle J x_{2}-x_{2}^{*}, x_{1}-x_{2}\right\rangle+\rho f\left(x_{1}\right)-\rho f\left(x_{2}\right) \geq 0 .
\end{aligned}
$$

It follows from (20) that

$$
\left\langle J x_{1}-x_{1}^{*}, x_{2}-x_{1}\right\rangle+\left\langle J x_{2}-x_{2}^{*}, x_{1}-x_{2}\right\rangle \geq 0
$$

and so

$$
\left\langle x_{1}^{*}-x_{2}^{*}, x_{1}-x_{2}\right\rangle \geq\left\langle J x_{1}-J x_{2}, x_{1}-x_{2}\right\rangle .
$$

By (19), we have

$$
\left\langle x_{1}^{*}-x_{2}^{*}, x_{1}-x_{2}\right\rangle \geq 2 C_{1}^{2} \delta_{B}\left(\frac{\left\|x_{1}-x_{2}\right\|}{2 C_{1}}\right),
$$

where $C_{1}=\sqrt{\left(\left\|x_{1}\right\|^{2}+\left\|x_{2}\right\|^{2}\right) / 2}$. This completes the proof.

Theorem 13. Let $B$ be a reflexive, smooth, and 2-uniformly convex Banach space with the dual space $B^{*}$ and $\delta(\epsilon) \geq$ $k_{B} \epsilon^{2}, \forall \epsilon \in(0,2]$ for some $k_{B}>0$. Then there exist a constant $K_{B}=\left(2 / k_{B}\right)$ such that

$$
\left\|\pi_{K}^{f} x_{1}^{*}-\pi_{K}^{f} x_{2}^{*}\right\| \leq K_{B}\left\|x_{1}^{*}-x_{2}^{*}\right\|, \quad \forall x_{1}^{*}, x_{2}^{*} \in B^{*} .
$$

Proof. From Proposition 12, it follows that

$$
\left\langle x_{1}^{*}-x_{2}^{*}, \pi_{K}^{f} x_{1}^{*}-\pi_{K}^{f} x_{2}^{*}\right\rangle \geq 2 C_{1}^{2} \delta\left(\frac{\left\|\pi_{K}^{f} x_{1}^{*}-\pi_{K}^{f} x_{2}^{*}\right\|}{2 C_{1}}\right),
$$


where $C_{1}=\sqrt{\left(\left\|\pi_{K}^{f} x_{1}^{*}\right\|^{2}+\left\|\pi_{K}^{f} x_{2}^{*}\right\|^{2}\right) / 2}$. Since $\delta_{B}(\epsilon) \geq k_{B} \epsilon^{2}$, (25) implies that

$$
\begin{aligned}
\left\langle x_{1}^{*}-x_{2}^{*}, \pi_{K}^{f} x_{1}^{*}-\pi_{K}^{f} x_{2}^{*}\right\rangle & \geq 2 C_{1}^{2} \delta_{B}\left(\frac{\left\|\pi_{K}^{f} x_{1}^{*}-\pi_{K}^{f} x_{2}^{*}\right\|}{2 C_{1}}\right) \\
& \geq \frac{k_{B}}{2}\left\|\pi_{K}^{f} x_{1}^{*}-\pi_{K}^{f} x_{2}^{*}\right\|^{2},
\end{aligned}
$$

and so

$$
\left\|\pi_{K}^{f} x_{1}^{*}-\pi_{K}^{f} x_{2}^{*}\right\| \leq \frac{2}{k_{B}}\left\|x_{1}^{*}-x_{2}^{*}\right\|=K_{B}\left\|x_{1}^{*}-x_{2}^{*}\right\| .
$$

This completes the proof.

Theorem 14. Let $B$ be a real 2-uniformly smooth and uniformly convex Banach space with the dual space $B^{*}$ and $\rho_{B}(t) \leq$ $c_{B} t^{2}$ for some $c_{B}>0$. Then there exist a constant $C=32 c_{B}>0$ such that

$$
\|J x-J y\| \leq C\|x-y\|, \quad \forall x, y \in B .
$$

Proof. From Proposition 11, it follows that

$$
\|J x-J y\| \leq 8 M_{2} h_{B}\left(\frac{4\|x-y\|}{M_{2}}\right), \quad x, y \in B,
$$

where $M_{2}=\sqrt{\left(\|x\|^{2}+\|y\|^{2}\right) / 2}$ and $h_{B}(\tau)=\rho_{B}(\tau) / \tau$. Since $\rho_{B}(t) \leq c_{B} t^{2}$

$$
\begin{aligned}
\|J x-J y\| & \leq 8 M_{2} h_{B}\left(\frac{4\|x-y\|}{M_{2}}\right) \\
& \leq 32 c_{B}\|x-y\|=C\|x-y\| .
\end{aligned}
$$

This completes the proof.

\section{Main Results}

From Theorem 7, we know that the following theorem holds.

Theorem 15. Let $B$ be a 2-uniformly smooth and 2-uniformly convex Banach space. $\left(x^{*}, y^{*}\right)$ is a solution of the SNMVIP (1) if and only if $\left(x^{*}, y^{*}\right) \in K \times K$ satisfying

$$
\begin{aligned}
& x^{*}=\pi_{K}^{f}\left[J\left(y^{*}\right)-\left(\rho_{1} A_{1}\left(y^{*}\right)+\rho_{1} S_{1}\left(y^{*}\right)\right)\right], \\
& y^{*}=\pi_{K}^{f}\left[J\left(x^{*}\right)-\left(\eta_{1} A_{2}\left(x^{*}\right)+\eta_{1} S_{2}\left(x^{*}\right)\right)\right] .
\end{aligned}
$$

In the sequel, we shall show the existence and uniqueness of solution for the problems (1), (2), and (3), respectively. Next, we construct some new iterative algorithms for the problems (1), (2), and (3). We also give the convergence analysis of the iterative sequences generated by the algorithms.

Theorem 16. Let $B$ be a 2-uniformly smooth and 2-uniformly convex Banach space with $\rho_{B}(t) \leq c_{B} t^{2}$ for some $c_{B}>0$ and $\delta(\epsilon) \geq k_{B} \epsilon^{2}, \forall \epsilon \in(0,2]$ for some $k_{B}>0$. Let $A_{1}, A_{2}, S_{1}, S_{2}$ : $K \rightarrow B^{*}$ be single-valued mappings. Let $J: B \rightarrow B^{*}$ and $J^{*}: B^{*} \rightarrow B$ be the normalized duality mapping, and let $f: K \subset B \rightarrow R \cup\{+\infty\}$ be a proper lower semicontinuous mapping. Suppose that the following conditions are satisfied:

(i) for each $i \in\{1,2\}, S_{i}$ is J-strongly monotone with constants $k_{i}$ and $s_{i}$-Lipschitz continuous;

(ii) for each $i \in\{1,2\}, A_{i}$ is $l_{i}$-Lipschitz continuous;

and that there exist constants $\rho_{1}, \eta_{1}>0$ satisfying

$$
\begin{aligned}
& K_{B}=\frac{2}{k_{B}}, \quad C=32 c_{B}, \\
& \Theta_{1}=K_{B}\left(\sqrt{C^{2}-2 \eta_{1} k_{2}+C_{B} \eta_{1}^{2} s_{2}^{2}}+\eta_{1} l_{2}\right)<1, \\
& \Theta_{2}=K_{B}\left(\sqrt{C^{2}-2 \rho_{1} k_{1}+\rho_{1}^{2} s_{1}^{2} C_{B}}+\rho_{1} l_{1}\right)<1 .
\end{aligned}
$$

Then SNMVIP (1) has a unique solution $\left(x^{*}, y^{*}\right)$.

Proof. First, we prove the existence of the solution. Define a mapping $F: K \rightarrow K$ as follows:

$F(x)$

$$
\begin{aligned}
=\pi_{K}^{f}[J & \left(\pi_{K}^{f}\left(J(x)-\eta_{1}\left(A_{2}(x)+S_{2}(x)\right)\right)\right)-\rho_{1}\left(A_{1}+S_{1}\right) \\
& \left.\times\left(\pi_{K}^{f}\left(J(x)-\eta_{1}\left(A_{2}(x)+S_{2}(x)\right)\right)\right)\right] .
\end{aligned}
$$

For any $x, y \in K$, by Theorem 13 , we know that there exists $K_{B}=\left(2 / k_{B}\right)>0$ such that

$$
\begin{aligned}
& \|F(x)-F(y)\| \\
& =\| \pi_{K}^{f}\left[J\left(\pi_{K}^{f}\left(J(x)-\eta_{1}\left(A_{2}(x)+S_{2}(x)\right)\right)\right)\right. \\
& \left.-\rho_{1}\left(A_{1}+S_{1}\right)\left(\pi_{K}^{f}\left(J(x)-\eta_{1}\left(A_{2}(x)+S_{2}(x)\right)\right)\right)\right] \\
& -\pi_{K}^{f}\left[J\left(\pi_{K}^{f}\left(J(y)-\eta_{1}\left(A_{2}(y)+S_{2}(y)\right)\right)\right)\right. \\
& -\rho_{1}\left(A_{1}+S_{1}\right) \\
& \left.\times\left(\pi_{K}^{f}\left(J(y)-\eta_{1}\left(A_{2}(y)+S_{2}(y)\right)\right)\right)\right] \| . \\
& \leq K_{B} \|\left[J\left(\pi_{K}^{f}\left(J(x)-\eta_{1}\left(A_{2}(x)+S_{2}(x)\right)\right)\right)\right. \\
& -J\left(\pi_{K}^{f}\left(J(y)-\eta_{1}\left(A_{2}(y)+S_{2}(y)\right)\right)\right) \\
& -\rho_{1}\left(S_{1}\left(\pi_{K}^{f}\left(J(x)-\eta_{1}\left(A_{2}(x)+S_{2}(x)\right)\right)\right)\right. \\
& \left.\left.-S_{1}\left(\pi_{K}^{f}\left(J(y)-\eta_{1}\left(A_{2}(y)+S_{2}(y)\right)\right)\right)\right)\right] \| \\
& +\rho_{1} \| A_{1}\left(\pi_{K}^{f}\left(J(x)-\eta_{1}\left(A_{2}(x)+S_{2}(x)\right)\right)\right) \\
& -A_{1}\left(\pi_{K}^{f}\left(J(y)-\eta_{1}\left(A_{2}(y)+S_{2}(y)\right)\right)\right) \| .
\end{aligned}
$$


Theorem 14 implies that there exists $C=32 c_{B}>0$ such that

$$
\|J(x)-J(y)\| \leq C\|x-y\|, \quad \forall x, y \in K .
$$

From condition (i) and Lemma 8, it follows that

$$
\begin{gathered}
\| J\left(\pi_{K}^{f}\left(J(x)-\eta_{1}\left(A_{2}(x)+S_{2}(x)\right)\right)\right) \\
-J\left(\pi_{K}^{f}\left(J(y)-\eta_{1}\left(A_{2}(y)+S_{2}(y)\right)\right)\right) \\
-\rho_{1}\left(S_{1}\left(\pi_{K}^{f}\left(J(x)-\eta_{1}\left(A_{2}(x)+S_{2}(x)\right)\right)\right)\right. \\
\left.-S_{1}\left(\pi_{K}^{f}\left(J(y)-\eta_{1}\left(A_{2}(y)+S_{2}(y)\right)\right)\right)\right) \|^{2} \\
=\|\left(\pi_{K}^{f}\left(J(x)-\eta_{1}\left(A_{2}(x)+S_{2}(x)\right)\right)\right) \\
+J\left(\pi_{K}^{f}\left(J(y)-\eta_{1}\left(A_{2}(y)+S_{2}(y)\right)\right)\right) \|^{2} \\
+\rho_{1}^{2} C_{B} \| S_{1}\left(\pi_{K}^{f}\left(J(x)-\eta_{1}\left(A_{2}(x)+S_{2}(x)\right)\right)\right) \\
-S_{1}\left(\pi_{K}^{f}\left(J(y)-\eta_{1}\left(A_{2}(y)+S_{2}(y)\right)\right)\right) \|^{2} \\
-2 C_{K}^{f}\left(J(x)-\eta_{1}\left(A_{2}(x)+S_{2}(x)\right)\right) \\
\left.-2 \rho_{1} k_{1}+\rho_{1}^{2} s_{1}^{2} C_{B}\right) \\
-S_{1}\left(\pi_{K}^{f}\left(J(x)-\eta_{1}\left(A_{2}(x)+S_{2}(x)\right)\right)\right) \\
J^{*}\left(J\left(\pi_{K}^{f}\left(J(y)-\eta_{1}\left(A_{2}(y)+S_{2}(y)\right)\right)\right),\right.
\end{gathered}
$$

By condition (ii) and Theorem 13, we know that

$$
\begin{gathered}
\| \pi_{K}^{f}\left(J(x)-\eta_{1}\left(A_{2}(x)+S_{2}(x)\right)\right) \\
-\pi_{K}^{f}\left(J(y)-\eta_{1}\left(A_{2}(y)+S_{2}(y)\right)\right) \| . \\
\leq K_{B}\left[\left\|J(x)-J(y)-\eta_{1}\left(S_{2}(x)-S_{2}(y)\right)\right\|\right. \\
\left.\quad+\eta_{1}\left\|A_{2}(x)-A_{2}(y)\right\|\right] \\
\leq K_{B}\left[\left\|J(x)-J(y)-\eta_{1}\left(S_{2}(x)-S_{2}(y)\right)\right\|\right. \\
\left.+\eta_{1} l_{2}\|x-y\|\right] .
\end{gathered}
$$

By (35), condition (i), and Lemma 8, we have

$$
\begin{aligned}
\left\|J(x)-J(y)-\eta_{1}\left(S_{2}(x)-S_{2}(y)\right)\right\|^{2} \\
\leq\|J(x)-J(y)\|^{2}-2 \eta_{1} \\
\quad \times\left\langle S_{2}(x)-S_{2}(y), J^{*}(J(x)-J(y))\right\rangle \\
\quad+C_{B} \eta_{1}^{2}\left\|S_{2}(x)-S_{2}(y)\right\|^{2} \\
\leq C^{2}\|x-y\|^{2}-2 \eta_{1} k_{2}\|x-y\|_{1}^{2}+C_{B} \eta_{1}^{2} s_{2}^{2}\|x-y\|^{2} \\
=\left(C^{2}-2 \eta_{1} k_{2}+C_{B} \eta_{1}^{2} s_{2}^{2}\right)\|x-y\|^{2} .
\end{aligned}
$$

Thus

$$
\begin{gathered}
\| \pi_{K}^{f}\left(J(x)-\eta_{1}\left(A_{2}(x)+S_{2}(x)\right)\right) \\
-\pi_{K}^{f}\left(J(y)-\eta_{1}\left(A_{2}(y)+S_{2}(y)\right)\right) \| \\
\leq K_{B}\left[\left\|J(x)-J(y)-\eta_{1}\left(S_{2}(x)-S_{2}(y)\right)\right\|\right. \\
\left.+\eta_{1} l_{2}\|x-y\|\right] \\
\leq K_{B}\left(\sqrt{C^{2}-2 \eta_{1} k_{2}+C_{B} \eta_{1}^{2} s_{2}^{2}}+\eta_{1} l_{2}\right)\|x-y\| .
\end{gathered}
$$

Condition (ii) and (39) imply that

$$
\begin{gathered}
\| A_{1}\left(\pi_{K}^{f}\left(J(x)-\eta_{1}\left(A_{2}(x)+S_{2}(x)\right)\right)\right) \\
-A_{1}\left(\pi_{K}^{f}\left(J(y)-\eta_{1}\left(A_{2}(y)+S_{2}(y)\right)\right)\right) \| \\
\leq l_{1} \| \pi_{K}^{f}\left(J(x)-\eta_{1}\left(A_{2}(x)+S_{2}(x)\right)\right) \\
-\pi_{K}^{f}\left(J(y)-\eta_{1}\left(A_{2}(y)+S_{2}(y)\right)\right) \| \\
\leq l_{1} K_{B}\left(\sqrt{C^{2}-2 \eta_{1} k_{2}+C_{B} \eta_{1}^{2} s_{2}^{2}}+\eta_{1} l_{2}\right)\|x-y\| .
\end{gathered}
$$

From (34)-(40), we have

$$
\begin{aligned}
\| F(x) & -F(y) \| \\
\leq & K_{B}^{2}\left(\sqrt{C^{2}-2 \eta_{1} k_{2}+C_{B} \eta_{1}^{2} s_{2}^{2}}+\eta_{1} l_{2}\right) \\
& \times\left[\sqrt{C^{2}-2 \rho_{1} k_{1}+\rho_{1}^{2} s_{1}^{2} C_{B}}+\rho_{1} l_{1}\right]\|x-y\| \\
= & \Theta_{1} \Theta_{2}\|x-y\| .
\end{aligned}
$$

It follows from (32) that $0<\Theta_{1}<1$ and $0<\Theta_{2}<1$. Thus, (41) implies that $F$ is a contractive mapping and so there exists a point $x^{*} \in K$ such that $x^{*}=F\left(x^{*}\right)$. Let

$$
y^{*}=\pi_{K}^{f}\left[J\left(x^{*}\right)-\left(\eta_{1} A_{2}\left(x^{*}\right)+\eta_{1} S_{2}\left(x^{*}\right)\right)\right] .
$$

From the definition of $F$, we have

$$
\begin{aligned}
& x^{*}=\pi_{K}^{f}\left[J\left(y^{*}\right)-\left(\rho_{1} A_{1}\left(y^{*}\right)+\rho_{1} S_{1}\left(y^{*}\right)\right)\right], \\
& y^{*}=\pi_{K}^{f}\left[J\left(x^{*}\right)-\left(\eta_{1} A_{2}\left(x^{*}\right)+\eta_{1} S_{2}\left(x^{*}\right)\right)\right] .
\end{aligned}
$$


By Theorem 15, we know that $\left(x^{*}, y^{*}\right)$ is a solution of SNMVIP (1).

Next, we show the uniqueness of the solution. Let $\left(u^{*} v^{*}\right)$ be another solution of SNMVIP (1). It follows from Theorem 15 that

$$
\begin{aligned}
& u^{*}=\pi_{K}^{f}\left[J\left(v^{*}\right)-\left(\rho_{1} A_{1}\left(v^{*}\right)+\rho_{1} S_{1}\left(v^{*}\right)\right)\right], \\
& v^{*}=\pi_{K}^{f}\left[J\left(u^{*}\right)-\left(\eta_{1} A_{2}\left(u^{*}\right)+\eta_{1} S_{2}\left(u^{*}\right)\right)\right] .
\end{aligned}
$$

As the proof of (41), we have

$$
\left\|x^{*}-u^{*}\right\| \leq \Theta_{1} \Theta_{2}\left\|x^{*}-u^{*}\right\| .
$$

Since $0<\Theta_{1}<1$ and $0<\Theta_{2}<1$, it follows that $u^{*}=x^{*}$ and so $v^{*}=y^{*}$. This completes the proof.

It is clear that if $B$ is a Hilbert space, then we can take $K_{B}=C=C_{B}=1$ in Theorem 16. According to Theorem 16, it is easy to get the following corollaries.

Corollary 17. Let $H$ be a real Hilbert space, let $K \subset H$ be a nonempty closed convex set, and let $S: K \rightarrow H$ be strongly monotone with constants $k$ and s-Lipschitz continuous. There exist $\rho_{1}, \eta_{1}>0$ satisfying

$$
\begin{aligned}
& \sqrt{1-2 \eta_{1} k+\eta_{1}^{2} s^{2}}<1, \\
& \sqrt{1-2 \rho_{1} k+\rho_{1}^{2} s^{2}}<1 .
\end{aligned}
$$

Then the problem (2) has a unique solution $\left(x^{*}, y^{*}\right)$.

Corollary 18. Let $H$ be a real Hilbert space, let $K \subset H$ be a nonempty closed convex set, and let $S: K \rightarrow H$ be strongly monotone with constants $k$ and s-Lipschitz continuous. There exists $\eta_{1}>0$ satisfying

$$
\sqrt{1-2 \eta_{1} k+\eta_{1}^{2} s^{2}}<1
$$

Then the problem (3) has a unique solution $\left(x^{*}, y^{*}\right)$.

Algorithm 19. For any given $x_{0} \in K$, define the Mann iterative sequences $\left\{x_{n}\right\}$ and $\left\{y_{n}\right\}$ with errors as follows:

$$
\begin{aligned}
& x_{n+1}=\left(1-\alpha_{n}\right) x_{n}+\alpha_{n} \pi_{K}^{f} \\
& \times\left[J\left(y_{n}\right)-\rho_{1}\left(A_{1}\left(y_{n}\right)+S_{1}\left(y_{n}\right)\right)\right]+\alpha_{n} u_{n}+w_{n}, \\
& y_{n}=\pi_{K}^{f}\left[J\left(x_{n}\right)-\eta_{1}\left(A_{2}\left(x_{n}\right)+S_{2}\left(x_{n}\right)\right)\right]+v_{n},
\end{aligned}
$$

where $\left\{\alpha_{n}\right\}$ is a sequence in $[0,1]$ and $\left\{u_{n}\right\},\left\{v_{n}\right\}$, and $\left\{w_{n}\right\}$ are three sequences in $B$ satisfying the following conditions:

$$
\begin{gathered}
\sum_{n=0}^{\infty} \alpha_{n}=+\infty, \quad \lim _{n \rightarrow \infty} \sum_{n=0}^{\infty}\left\|w_{n}\right\|<+\infty, \\
\lim _{n \rightarrow \infty}\left\|u_{n}\right\|=\lim _{n \rightarrow \infty}\left\|v_{n}\right\|=0 .
\end{gathered}
$$

If $B=H$ is a real Hilbert space, $S_{1}=S_{2}=S, A_{1}=A_{2}=$ 0 , and $f=0$, then Algorithm 19 reduces to the following iterative algorithm.

Algorithm 20 (see [2, Algorithm 2.1]). For any given $x_{0} \in K$, define the iterative sequences $\left\{x_{n}\right\}$ and $\left\{y_{n}\right\}$ as follows:

$$
\begin{gathered}
x_{n+1}=\left(1-\alpha_{n}\right) x_{n}+\alpha_{n} P_{K}\left[y_{n}-\rho_{1} S\left(y_{n}\right)\right], \\
y_{n}=P_{K}\left[x_{n}-\eta_{1} S\left(x_{n}\right)\right],
\end{gathered}
$$

where $\left\{\alpha_{n}\right\}$ is a sequence in $[0,1]$ satisfying the following conditions:

$$
\sum_{n=0}^{\infty} \alpha_{n}=+\infty .
$$

If $B=H$ is a real Hilbert space, $S_{1}=S_{2}=S, A_{1}=A_{2}=0$, $\eta_{1}=0$, and $f=0$, then Algorithm 19 reduces to the following iterative algorithm.

Algorithm 21 (see [1, Algorithm 2.2]). For any given $x_{0} \in K$, define the iterative sequences $\left\{x_{n}\right\}$ as follows:

$$
x_{n+1}=\left(1-\alpha_{n}\right) x_{n}+\alpha_{n} P_{K}\left[x_{n}-\eta_{1} S\left(x_{n}\right)\right],
$$

where $\left\{\alpha_{n}\right\}$ is a sequence in $[0,1]$ satisfying the following conditions:

$$
\sum_{n=0}^{\infty} \alpha_{n}=+\infty
$$

Theorem 22. Let $A_{1}, A_{2}, S_{1}, S_{2}, J$, and $J^{*}$ be the same as in Theorem 16, and let $\left\{x_{n}, y_{n}\right\}$ be the iterative sequence generated by Algorithm 19. If the condition (32) holds, then $\left(x_{n}, y_{n}\right)$ converges strongly to the unique solution $\left(x^{*} ; y^{*}\right)$ of SNMVIP (1).

Proof. By Theorem 16, we know that SNMVIP (1) has a unique solution $\left(x^{*} ; y^{*}\right)$. It follows from Theorem 15 that

$$
\begin{aligned}
& x^{*}=\pi_{K}^{f}\left[J\left(y^{*}\right)-\left(\rho_{1} A_{1}\left(y^{*}\right)+\rho_{1} S_{1}\left(y^{*}\right)\right)\right], \\
& y^{*}=\pi_{K}^{f}\left[J\left(x^{*}\right)-\left(\eta_{1} A_{2}\left(x^{*}\right)+\eta_{1} S_{2}\left(x^{*}\right)\right)\right] .
\end{aligned}
$$

By Lipschitz property of $A_{1}$, Theorem 13, (48), and (54), we have

$$
\begin{aligned}
\left\|x_{n+1}-x^{*}\right\|=\| & \left(1-\alpha_{n}\right) x_{n}+\alpha_{n} \pi_{K}^{f} \\
& \times\left[J\left(y_{n}\right)-\rho_{1}\left(A_{1}\left(y_{n}\right)+S_{1}\left(y_{n}\right)\right)\right] \\
& +\alpha_{n} u_{n}+w_{n}-x^{*} \| \\
\leq & \left(1-\alpha_{n}\right)\left\|x_{n}-x^{*}\right\| \\
& +\alpha_{n} \| \pi_{K}^{f}\left[J\left(y_{n}\right)-\rho_{1}\left(A_{1}\left(y_{n}\right)+S_{1}\left(y_{n}\right)\right)\right] \\
& -\pi_{K}^{f}\left[J\left(y^{*}\right)-\left(\rho_{1} A_{1}\left(y^{*}\right)+\rho_{1} S_{1}\left(y^{*}\right)\right)\right] \| \\
& +\alpha_{n}\left\|u_{n}\right\|+\left\|w_{n}\right\| \leq\left(1-\alpha_{n}\right)\left\|x_{n}-x^{*}\right\|
\end{aligned}
$$




$$
\begin{aligned}
& +\alpha_{n} K_{B} \| J\left(y_{n}\right)-J\left(y^{*}\right) \\
& -\rho_{1}\left(S_{1}\left(y_{n}\right)-S_{1}\left(y^{*}\right)\right) \\
& \quad-\rho_{1}\left(A_{1}\left(y_{n}\right)-A_{1}\left(y^{*}\right)\right) \| \\
& +\alpha_{n}\left\|u_{n}\right\|+\left\|w_{n}\right\| \\
& \leq\left(1-\alpha_{n}\right)\left\|x_{n}-x^{*}\right\|+\alpha_{n} K_{B} \\
& \times\left\|J\left(y_{n}\right)-J\left(y^{*}\right)-\rho_{1}\left(S_{1}\left(y_{n}\right)-S_{1}\left(y^{*}\right)\right)\right\| \\
& +K_{B} \rho_{1} l_{1}\left\|y_{n}-y^{*}\right\|+\alpha_{n}\left\|u_{n}\right\|+\left\|w_{n}\right\| . \quad(55)
\end{aligned}
$$

Since $S_{1}$ is $J$-strongly monotone with constants $k_{1}$ and $s_{1}$-Lipschitz continuous, by Theorem 14 and Lemma 8 , we obtain

$$
\begin{aligned}
& \left\|J\left(y_{n}\right)-J\left(y^{*}\right)-\rho_{1}\left(S_{1}\left(y_{n}\right)-S_{1}\left(y^{*}\right)\right)\right\| \\
& \leq \sqrt{C^{2}-2 \rho_{1} k_{1}+C_{B} \rho_{1}^{2} s_{1}^{2}}\left\|y_{n}-y^{*}\right\| .
\end{aligned}
$$

Combining (55) and (56), we obtain

$$
\begin{aligned}
& \left\|x_{n+1}-x^{*}\right\| \leq\left(1-\alpha_{n}\right)\left\|x_{n}-x^{*}\right\| \\
& +\alpha_{n} \Theta_{2}\left\|y_{n}-y^{*}\right\|+\alpha_{n}\left\|u_{n}\right\|+\left\|w_{n}\right\| .
\end{aligned}
$$
that

From Lipschitz property of $A_{2}$, (48), and (54), it follows

$$
\begin{aligned}
& \left\|y_{n}-y^{*}\right\| \\
& =\| \pi_{K}^{f}\left[J\left(x_{n}\right)-\eta_{1}\left(A_{2}\left(x_{n}\right)+S_{2}\left(x_{n}\right)\right)\right] \\
& \quad+v_{n}-\pi_{K}^{f}\left[J\left(x^{*}\right)-\left(\eta_{1} A_{2}\left(x^{*}\right)+\eta_{1} S_{2}\left(x^{*}\right)\right)\right] \| \\
& \leq K_{B} \| J\left(x_{n}\right)-J\left(x^{*}\right)-\eta_{1}\left(S_{2}\left(x_{n}\right)-S_{2}\left(x^{*}\right)\right) \\
& \quad-\eta_{1}\left(A_{2}\left(x_{n}\right)-A_{2}\left(x^{*}\right)\right)\|+\| v_{n} \| \\
& \leq K_{B}\left\|J\left(x_{n}\right)-J\left(x^{*}\right)-\eta_{1}\left(S_{2}\left(x_{n}\right)-S_{2}\left(x^{*}\right)\right)\right\| \\
& \quad+\left\|v_{n}\right\|+K_{B} \eta_{1} l_{2}\left\|x_{n}-x^{*}\right\| .
\end{aligned}
$$

Since $S_{2}$ is $J$-strongly monotone with constants $k_{2}$ and $s_{2}$ Lipschitz continuous, Theorem 14 and Lemma 8 imply that

$$
\begin{gathered}
\left\|J\left(x_{n}\right)-J\left(x^{*}\right)-\eta_{1}\left(S_{2}\left(x_{n}\right)-S_{2}\left(x^{*}\right)\right)\right\| \\
\leq \sqrt{C^{2}-2 \eta_{1} k_{2}+C_{B} \eta_{1}^{2} s_{2}^{2}}\left\|x_{n}-x^{*}\right\| .
\end{gathered}
$$

It follows from (57)-(59) that

$$
\begin{aligned}
& \left\|x_{n+1}-x^{*}\right\| \\
& \leq\left(1-\alpha_{n}\right)\left\|x_{n}-x^{*}\right\|+\alpha_{n} \Theta_{2} \Theta_{1}\left\|x_{n}-x^{*}\right\| \\
& \quad+\alpha_{n} \Theta_{2}\left\|v_{n}\right\|+\alpha_{n}\left\|u_{n}\right\|+\left\|w_{n}\right\| \\
& =\left(1-\alpha_{n}\left(1-\Theta_{2} \Theta_{1}\right)\right)\left\|x_{n}-x^{*}\right\|+\left\|w_{n}\right\| \\
& \quad+\alpha_{n}\left(1-\Theta_{2} \Theta_{1}\right) \frac{1}{\left(1-\Theta_{2} \Theta_{1}\right)}\left(\Theta_{2}\left\|v_{n}\right\|+\left\|u_{n}\right\|\right) .
\end{aligned}
$$

Taking $t_{n}=\alpha_{n}\left(1-\Theta_{2} \Theta_{1}\right), a_{n}=\left\|x_{n+1}-x^{*}\right\|, b_{n}=1 /(1-$ $\left.\Theta_{2} \Theta_{1}\right)\left(\Theta_{2}\left\|v_{n}\right\|+\left\|u_{n}\right\|\right)$, and $c_{n}=\left\|w_{n}\right\|$, Lemma 9 implies that $a_{n} \rightarrow 0$ and so $x_{n} \rightarrow x^{*}$ as $n \rightarrow \infty$. Since $x_{n} \rightarrow x^{*}$, by (58) and (59), we know that $y_{n} \rightarrow y^{*}$ as $n \rightarrow \infty$. This completes the proof.

Corollary 23 (see [2, Theorem 2.1]). Let H, K, and $S$ be the same as in Corollary 17, and let $\left\{x_{n}\right\}$ and $\left\{y_{n}\right\}$ be the iterative sequence generated by Algorithm 20. If there exist constants $\rho_{1}, \eta_{1}>0$ satisfying

$$
\begin{aligned}
& \sqrt{1-2 \eta_{1} k+\eta_{1}^{2} s^{2}}<1, \\
& \sqrt{1-2 \rho_{1} k+\rho_{1}^{2} s^{2}}<1,
\end{aligned}
$$

then $\left(x_{n}, y_{n}\right)$ converges strongly to the unique solution $\left(x^{*}, y^{*}\right)$ of the problem (2).

Corollary 24 (see [1, Theorem 3.3]). Let $H, K$, and $S$ be the same as in Corollary 18, and let $\left\{x_{n}\right\}$ be the iterative sequence generated by Algorithm 21. If there exists $\eta_{1}>0$ satisfying

$$
\sqrt{1-2 \eta_{1} k+\eta_{1}^{2} s^{2}}<1
$$

then $x_{n}$ converges strongly to the unique solution $x^{*}$ of the problem (3).

\section{Conflict of Interests}

The authors declare that there is no conflict of interests regarding the publication of this paper.

\section{Acknowledgments}

The second author was partially supported by the Guangxi Natural Science Foundation (2013GXNSFBA019015), the Scientific Research Foundation of Guangxi University for Nationalities (2012QD015), and the Open fund of Guangxi key laboratory of hybrid computation and IC design analysis (2013HCIC06). The third author was partially supported by the Key Program of the Fundamental Research Funds for the Central Universities (A0920502051202-111).

\section{References}

[1] R. U. Verma, "General convergence analysis for two-step projection methods and applications to variational problems," Applied Mathematics Letters, vol. 18, no. 11, pp. 1286-1292, 2005.

[2] R. U. Verma, "Projection methods, algorithms, and a new system of nonlinear variational inequalities," Computers \& Mathematics with Applications, vol. 41, no. 7-8, pp. 1025-1031, 2001.

[3] Y. Alber, "The regularization method for variational inequalities with nonsmooth unbounded operators in Banach space," Applied Mathematics Letters, vol. 6, no. 4, pp. 63-68, 1993.

[4] Y. Alber, "Metric and generalized projection operators in Banach spaces: properties and applications," in Theory and Applications of Nonlinear Operators of Accretive and Monotone Type, A. Kartsatos, Ed., vol. 178, pp. 15-50, Dekker, New York, NY, USA, 1996. 
[5] F. Facchinei and J. S. Pang, Finite Dimensional Variational Inequalities and Complementarity Problems, Springer, New York, NY, USA, 2003.

[6] J. H. Fan, X. Liu, and J. Li, "Iterative schemes for approximating solutions of generalized variational inequalities in Banach spaces," Nonlinear Analysis, vol. 70, no. 11, pp. 3997-4007, 2009.

[7] J. L. Li, "The generalized projection operator on reflexive Banach spaces and its applications," Journal of Mathematical Analysis and Applications, vol. 306, no. 1, pp. 55-71, 2005.

[8] X. Li, N. J. Huang, and D. O'Regan, “Strong convergence theorems for relatively nonexpansive mappings in Banach spaces with applications," Computers \& Mathematics with Applications, vol. 60 , no. 5, pp. 1322-1331, 2010.

[9] X. Li, N. J. Huang, and Y. Z. Zou, "On the stability of generalized $f$-projection operators with an application," Acta Mathematica Sinica, vol. 54, no. 5, pp. 811-822, 2011.

[10] K. Q. Wu and N. J. Huang, "The generalised $f$-projection operator with an application," Bulletin of the Australian Mathematical Society, vol. 73, no. 2, pp. 307-317, 2006.

[11] K. Q. Wu and N. J. Huang, "Properties of the generalized $f$-projection operator and its applications in Banach spaces," Computers \& Mathematics with Applications, vol. 54, no. 3, pp. 399-406, 2007.

[12] K. Q. Wu and N. J. Huang, "The generalized $f$-projection operator and set-valued variational inequalities in Banach spaces," Nonlinear Analysis, vol. 71, no. 7-8, pp. 2481-2490, 2009.

[13] H. K. Xu, "Inequalities in Banach spaces with applications," Nonlinear Analysis, vol. 16, no. 12, pp. 1127-1138, 1991.

[14] Y. Alber and J. C. Yao, "Another version of the proximal point algorithm in a Banach space," Nonlinear Analysis, vol. 70, no. 9, pp. 3159-3171, 2009.

[15] S. S. Chang, H. W. J. Lee, and C. K. Chan, "Generalized system for relaxed cocoercive variational inequalities in Hilbert spaces," Applied Mathematics Letters, vol. 20, no. 3, pp. 329-334, 2007.

[16] F. Q. Xia and N. J. Huang, "An inexact hybrid projectionproximal point algorithm for solving generalized mixed variational inequalities," Computers \& Mathematics with Applications, vol. 62, no. 12, pp. 4596-4604, 2011.

[17] F. Q. Xia and N. J. Huang, "A projection-proximal point algorithm for solving generalized variational inequalities," Journal of Optimization Theory and Applications, vol. 150, no. 1, pp. 98117, 2011.

[18] I. Cioranescu, Geometry of Banach Spaces, Duality Mappings and Nonlinear Problems, vol. 62, Kluwer Academic Publishers, Dordrecht, The Netherlands, 1990.

[19] W. Takahashi, Nonlinear Functional Analysis, Yokohama Publishers, Yokohama, Japan, 2000.

[20] J. K. Kim and D. S. Kim, "A new system of generalized nonlinear mixed variational inequalities in Hilbert spaces," Journal of Convex Analysis, vol. 11, no. 1, pp. 235-243, 2004.

[21] Y. H. Yao, Y. C. Liou, and S. M. Kang, “Two-step projection methods for a system of variational inequality problems in Banach spaces," Journal of Global Optimization, vol. 55, no. 4, pp. 801-811, 2013. 


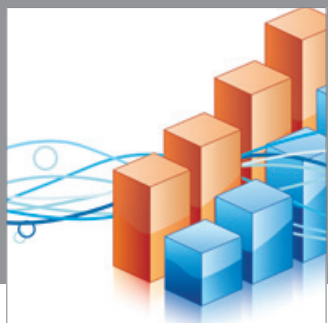

Advances in

Operations Research

mansans

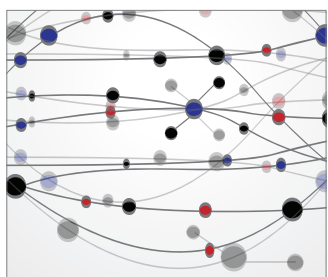

The Scientific World Journal
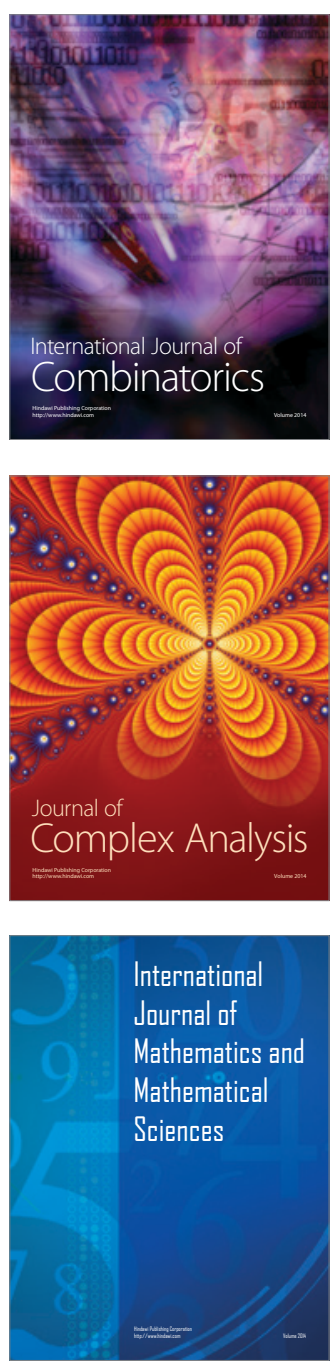
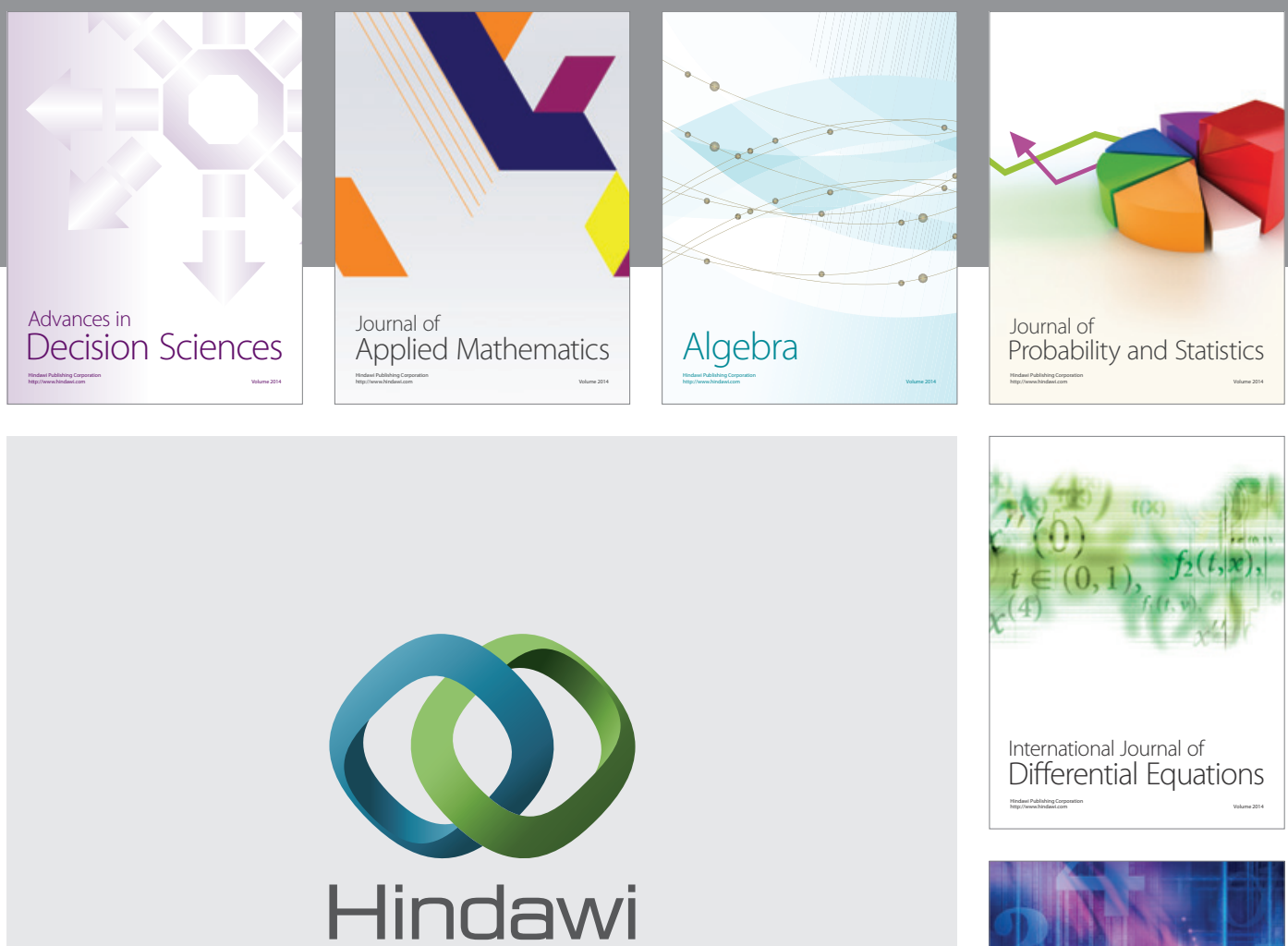

Submit your manuscripts at http://www.hindawi.com
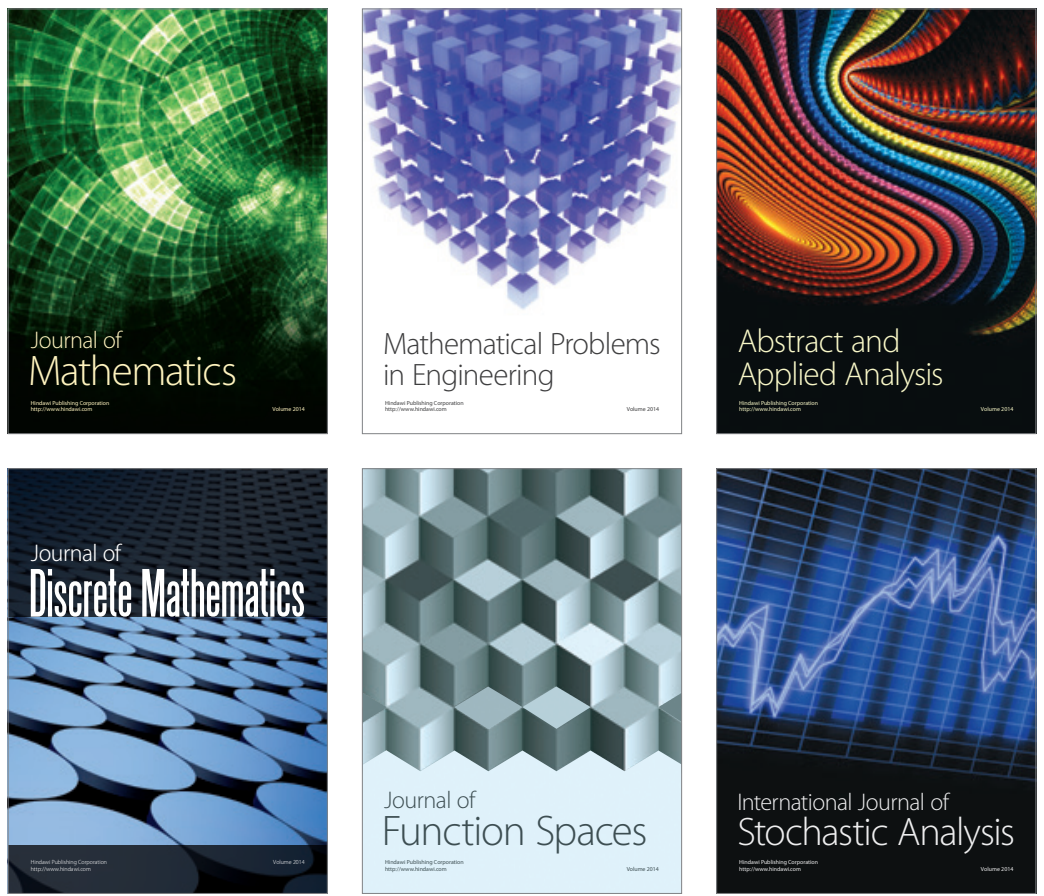

Journal of

Function Spaces

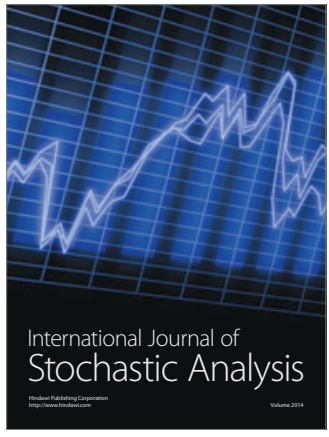

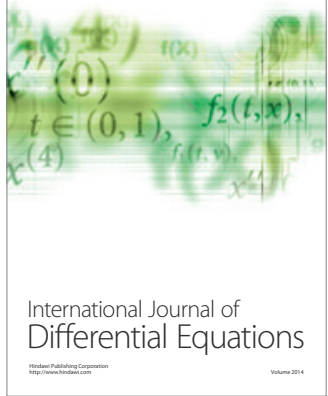
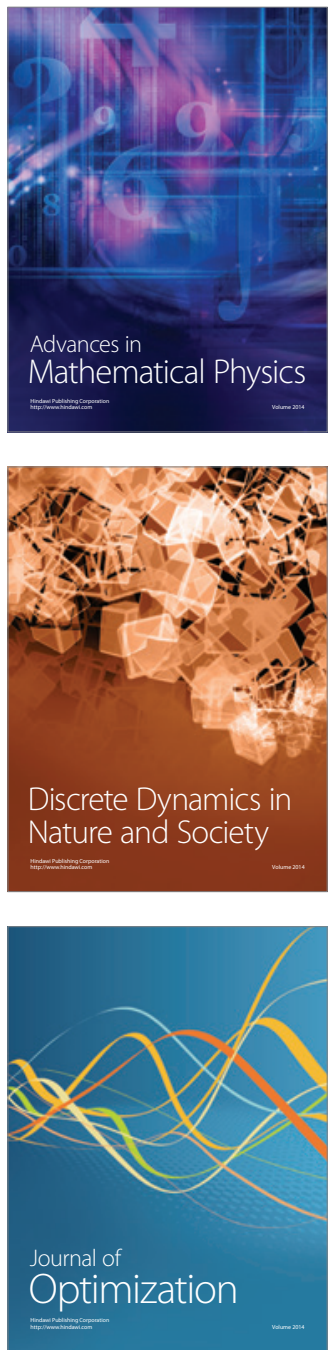